\title{
Prospecting for Genes involved in transcriptional regulation of plant defenses, a bioinformatics approach
}

\author{
Marcel C van Verk, John F Bol and Huub JM Linthorst
}

\begin{abstract}
Background: In order to comprehend the mechanisms of induced plant defense, knowledge of the biosynthesis and signaling pathways mediated by salicylic acid (SA), jasmonic acid (JA) and ethylene (ET) is essential. Potentially, many transcription factors could be involved in the regulation of these pathways, although finding them is a difficult endeavor. Here we report the use of publicly available Arabidopsis microarray datasets to generate gene co-expression networks.

Results: Using 372 publicly available microarray data sets, a network was constructed in which Arabidopsis genes for known components of SA, JA and ET pathways together with the genes of over 1400 transcription factors were assayed for co-expression. After determining the Pearson Correlation Coefficient cutoff to obtain the most probable biologically relevant co-expressed genes, the resulting network confirmed the presence of many genes previously reported in literature to be relevant for stress responses and connections that fit current models of stress gene regulation, indicating the potential of our approach. In addition, the derived network suggested new candidate genes and associations that are potentially interesting for future research to further unravel their involvement in responses to stress.
\end{abstract}

Conclusions: In this study large sets of stress related microarrays were used to reveal co-expression networks of transcription factors and signaling pathway components. These networks will benefit further characterization of the signal transduction pathways involved in plant defense.

Keywords: Co-expression analysis, salicylic acid-induced, jasmonic acid-induced, ethylene-induced, defense response, signal transduction, Arabidopsis, transcription factors

\section{Background}

Plants exposed to biotic and abiotic stress activate various signal transduction pathways, like the salicylic acid (SA)-, jasmonic acid (JA)-, ethylene (ET)-, and abscisic acid (ABA)-mediated signaling pathways that act singly or in combinations to evoke the most appropriate defense response [1-6]. For example, attack by pathogens results in extensive crosstalk between the SA-, JAand ET-signaling pathways, implicating complex regulatory networks underlying the plant's pathogen defense [3]. Arabidopsis contains almost 1500 genes encoding transcription factors [7] and it is safe to assume that

\footnotetext{
* Correspondence: h.j.m.linthorst@biology.leidenuniv.nl

Institute of Biology, Leiden University, Sylvius Laboratory, Sylviusweg 72,
} 2333 BE Leiden, The Netherlands many are involved in regulation of these defense-signaling pathways. However, the precise regulatory mechanisms and the transcription factors involved are mostly still unknown. To fine-tune the initiated defense responses the biosynthesis and signaling pathways influence each other via crosstalk. This makes discovery of novel regulatory elements within these pathways even more challenging.

The signaling that leads to defense proceeds via interactions of signaling pathway components and because of this, the genes involved are often expressed under similar conditions. This makes their expression cooperatively regulated and their expression patterns highly similar. Based on this concept, an analysis of co-regulated genes under a variety of conditions can give valuable information

\section{Biomed Central}


for understanding the possible regulatory mechanisms involved in defense responses. Any dataset consisting of at least two experiments can be used to perform a co-expression analysis, although for an analysis that is independent of the experimental conditions, a minimum of approximately 100 experiments is needed [8].

To investigate co-expressed genes in Arabidopsis many co-expression databases from different microarray sources with hundreds of experimental conditions per dataset have been developed in the last couple of years, such as Gene Expression Omnibus (http://www. ncbi.nlm.nih.gov/geo/[9]), ArrayExpress (http://www.ebi. ac.uk/microarray-as/ae/[10]), AthCor@CSB.DB (http:// csbdb.mpimp-golm.mpg.de[11]), Genevestigator (http:// www.genevestigator.com[12-14]), The Botany Array Resource (BAR; http://bbc.botany.utoronto.ca[15]), Arabidopsis Co-expression Data Mining Tool (ACT; http:// www.arabidopsis.leeds.ac.uk/act/[16]), ATTED-II (http:// atted.jp[17-19]), AtGenExpress/PRIME (http://prime.psc. riken.jp/[20]), and CressExpress (http://www.cressexpress.org[21]). Many of these databases only accept single-gene queries for analysis of a correlation coefficient. To obtain full flexibility in analysis method, data selection, filtering, etc., a more tailor made approach is needed. This can only be achieved after downloading the datasets and perform a manual analysis, which requires considerable computer power and knowledge about analysis methods, which is not essential for most of the available online tools.

Within the plant field there is an increasing number of publications that report the finding of biologically relevant genes involved in certain pathways via coexpression analysis. Examples are: genes involved in root development [22], genes involved in mitochondrial functions [23], clusters of genes involved in primary and secondary cell wall formation [24], Myb transcription factors responsible for initiation of aliphatic glucosinolate biosynthesis [25], and clusters of genes in a network related to cold stress and biochemical pathways [26]. In all these cases co-expression analysis assisted in building a network that linked unknown regulatory elements to already described pathways and helped expand hypotheses on how the genes in the network were regulated.

Although co-expression analysis tools are powerful in lead discovery, they cannot guarantee that observed coexpression of genes is biologically relevant. Further analysis using the 'classical' genomic and/or metabolomic approaches will still be necessary to confirm the involvement of the discovered genes. Despite this, co-expression analysis has proven itself as a very powerful tool in the discovery of new targets for analysis in pathways or networks of interest, as it can much more rapidly provide insight into potentially important network genes than random gain of function or loss of function approaches.
Here we report findings from a co-expression analysis covering a large number of microarray data sets derived from stress-induced Arabidopsis. In addition to genes already known to be involved in various stress-response pathways, a large number of new candidate genes were identified that potentially participate in regulation of stress-responses.

\section{Results and Discussion}

\section{Public Microarray Data Selection}

To discover new leads in the transcriptional regulation of the SA, JA and ET biosynthesis and signaling pathways under stress conditions an analysis of multiple transcriptome co-expression profiles was setup. For a flexible setup that is not limited to predefined settings, datasets or processing of samples, a dataset was downloaded from the TAIR website (ftp://ftp.arabidopsis.org/ Microarrays/analyzed_data/). This dataset consists of 1436 Affymetrix Arabidopsis 25K arrays obtained from NASCArrays and AtGenExpress. All microarrays were normalized by TAIR using the robust multi-array method (RMA).

To focus on stress-related SA, JA and ET biosynthesis and signaling pathways we performed a bi-clustering of all WRKY transcription factors spotted on the Affymetrix arrays versus a selected set of microarray data obtained from a variety of stress conditions. The stress data set of 372 microarrays as listed in Figure 1D was selected from the total of 1436 currently available microarrays. An overview of these 372 microarrays is given in Additional file 1, Table 1. For comparison, a set consisting of 237 development-related microarrays and a set consisting of all 1436 available microarrays were also analyzed. Hierarchical cluster trees with complete linkage and dendogram cutoffs of 0.50 were added for both the experimental conditions and the WRKY genes, and visualized using different colors. The result of this biclustering is shown in Figure 1A. The colors of the bar below the bi-clustering matrix correspond to the colored sets of arrays as denoted in Figure 1D. Similar bi-clusterings of WRKY gene expression profiles were performed with the subset of development-related microarrays and with the set containing all micro-arrays. The hierarchical cluster trees for the latter bi-clusterings are shown in Figures 1B and 1C, respectively.

It is evident that substantial differences occur in the hierarchical clustering of the WRKYs between the three sets of arrays. WRKY genes with coordinated expression patterns clustering close together under conditions of stress (Figure 1A) appeared not necessarily also co-regulated during development (Figure 1B). E.g., WRKYs 19 and 4 (Figure 1A, top) were clustered close together in the same sub-tree when the bi-clustering was done with the set of stress microarrays, but were situated far apart 


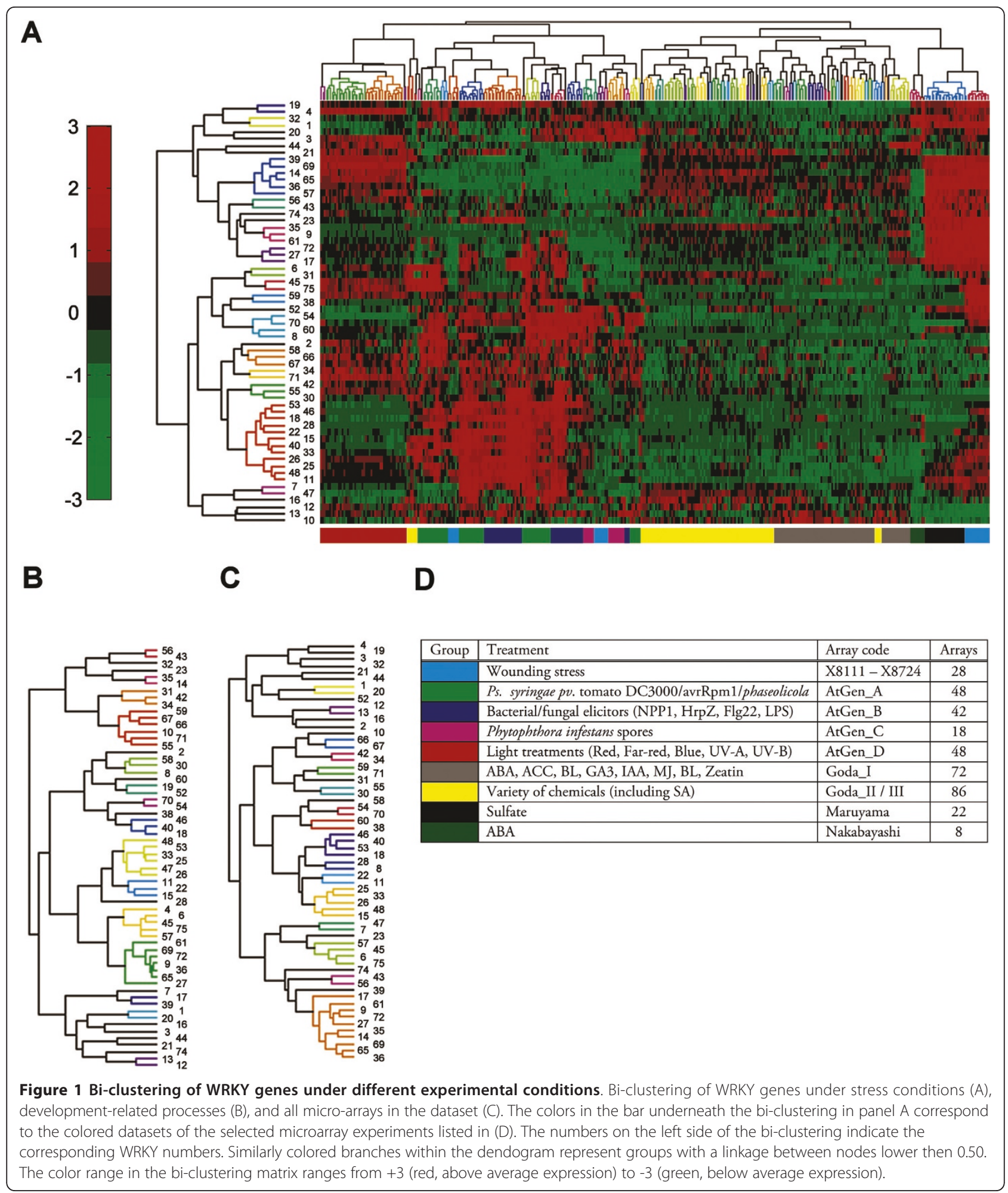

in separate sub-trees when the development-related arrays were used. The same is the case for WRKYs 28 and 46 (see below). Therefore, to maximize the probability that only biologically relevant correlations were obtained, we chose to use the dataset of the stressrelated microarrays listed in Figure 1D to investigate coexpression of genes involved in the SA, JA and ET pathways. 


\section{Target Gene Selection and Co-expression Cutoff Determination}

To elucidate new transcription factors regulating SA, JA and ET biosynthesis and signaling pathways we composed a set of genes consisting of all color-coded genes indicated in Figure 2. This set comprises many welldocumented genes attributed to the respective stresssignaling pathways [4]. This set was supplemented with a set of genes encoding almost 1400 transcription factors according to Czechowski et al. [7] and with the genes for the known JAZ repressor proteins and a number of other known regulators of these pathways. A listing of the genes in the set is given in Additional file 1, Table 1. To determine the Pearson Correlation Coefficient (PCC) cutoff for finding biologically relevant coexpressed genes and networks, various approaches can be applied. Several of these approaches are reviewed by Borate et al. [27] including maximal cliques, spectral graph clustering, correlation of control spots with expressed genes, top $1 \%$ of correlations, Bonferroni corrected $\mathrm{p}$-values, and statistical power. The first two methods resulted in the most biological reliable PCC cutoffs. Since a maximal cliques approach required more computational power than we had available and the spectral graph clustering easily results in cutoffs that are 0.05 off, we chose to apply the approach as described by Aoki et al. [8]. Their method, based on density of the network combined with decreasing number of nodes and edges with higher PCC values, closely approaches the biological relevant PCC and is easy to implement for biologists with modest computing power. The number of nodes (genes), edges (links between genes), the network density (a ratio of the observed number of edges to all possible edges), and the number of individual clusters obtained using the MCODE algorithm was determined for different PCC cutoffs using the genes listed in Additional file 1, Table 1 and Figure 2. The results are visualized in Figure 3A-D. The total number of nodes and edges increased with a decreasing PCC threshold (Figure 3A and 3B). In Figure 3A a linear increase in the number of nodes that have at least one link with another node is found between 0.62 and 0.82 . On the other hand, the number of edges below a cutoff of 0.70 starts to rapidly increase (Figure 3B), indicating that the available nodes become more densely connected as can also be seen with the increase in network density

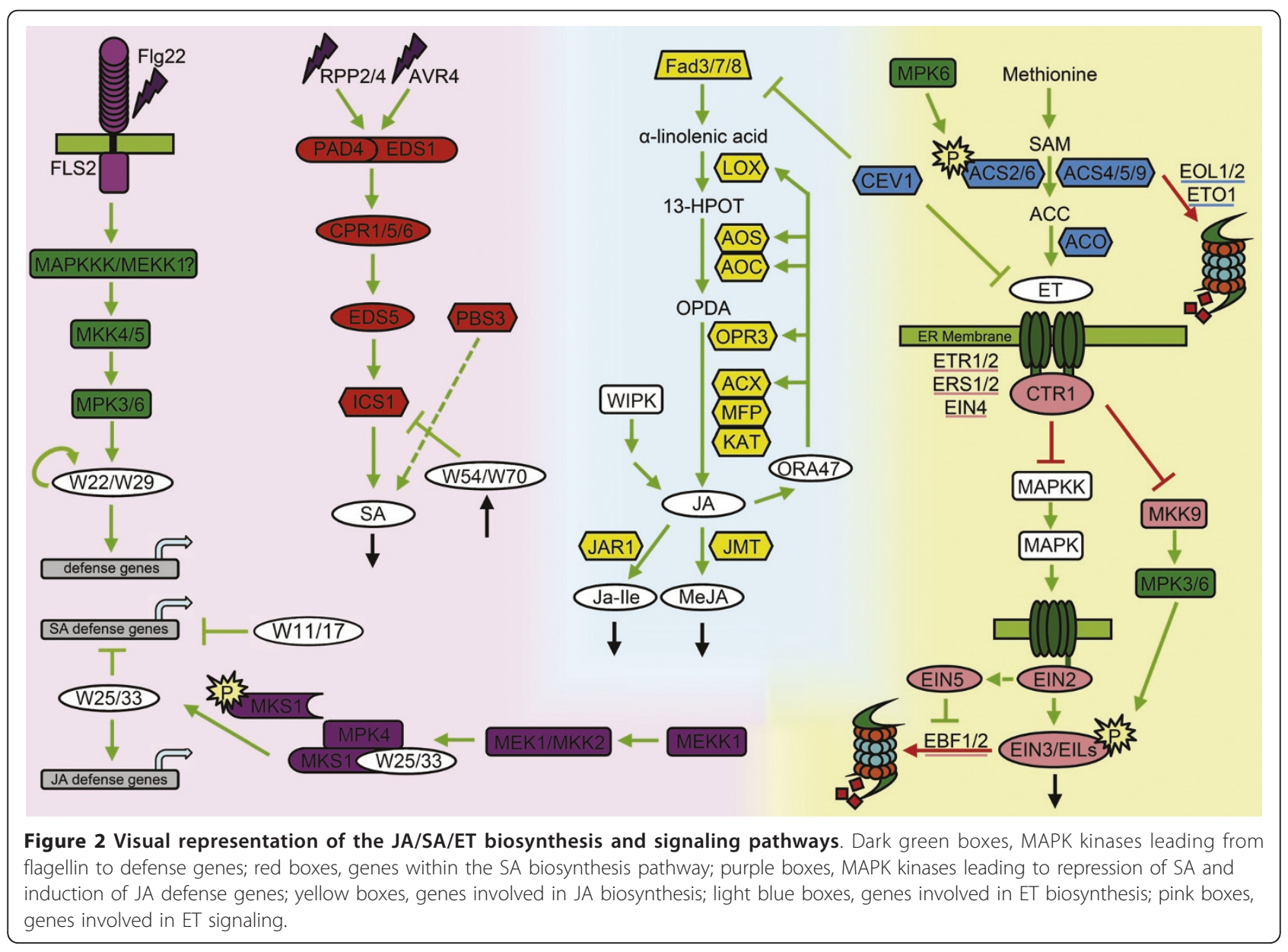



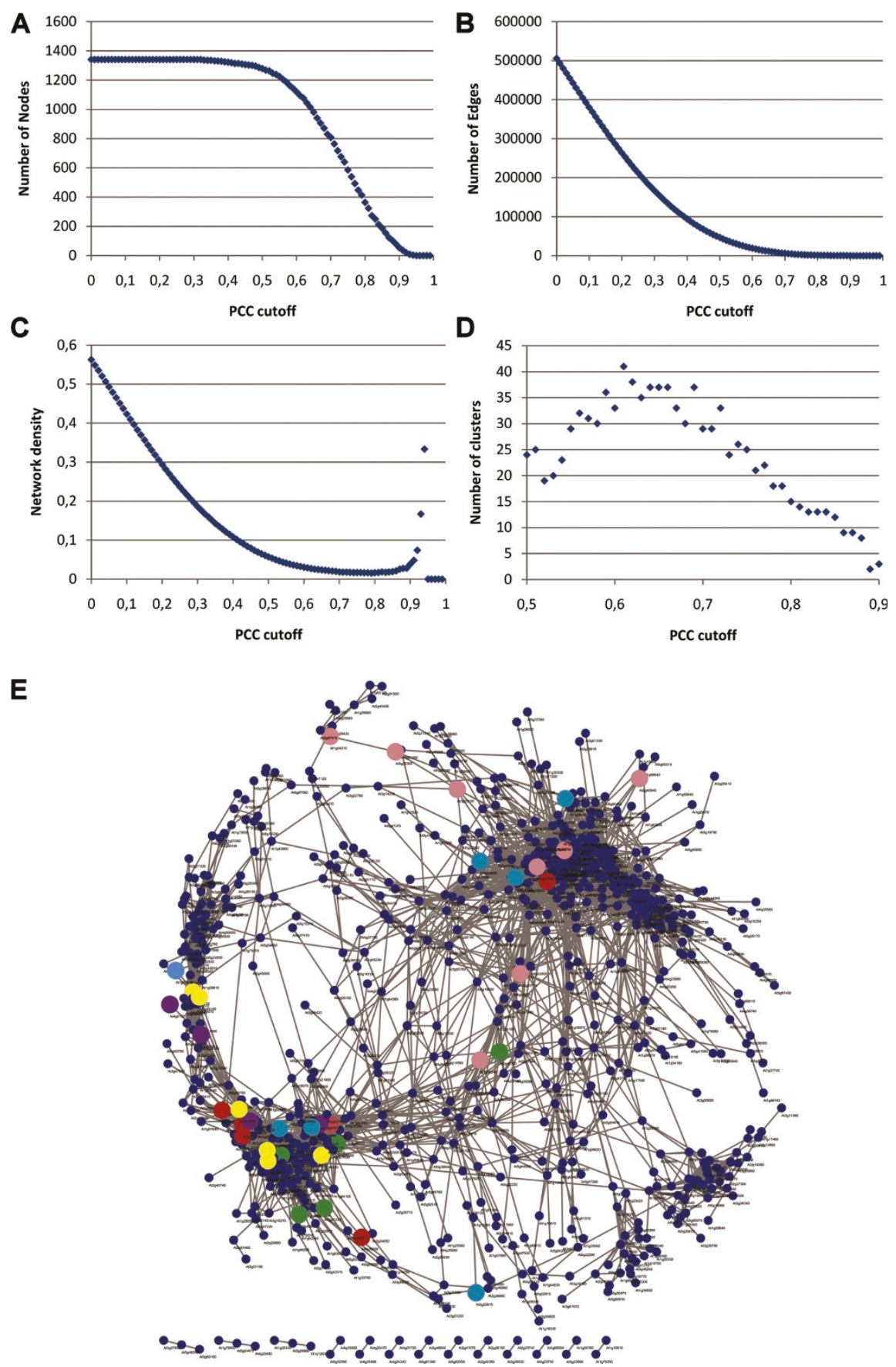

Figure 3 Pearson correlation coefficient cutoff determination and co-expression network. (A) Graph of the number of nodes with at least one link for each PCC cutoff. (B) Graph of the number of edges between nodes for each PCC cutoff. (C) Graph of the network density for each PCC cutoff. (D) Graph of the total number of clusters determined with the MCODE algorithm for each PCC cutoff. (E) Visualization using Cytoscape of the co-expression network. Blue-dots, on microarray spotted selection of $>1400$ transcription factors and JAZ proteins; other colored dots represent similarly colored genes from Figure 2 .

below this cutoff (Figure 3C). The region from 0.70 to 0.85 in Figure $3 \mathrm{C}$ indicates the minimum network density. According to the analysis of Aoki et al. [8] the most biological relevant PCC cutoff is found above these values. Combined, the data of Figure 3A-C leaves a relevant range for the cutoff between 0.70 to 0.82 . To evaluate the number of clusters related to this range of closely co-regulated genes inside the network, the 
MCODE algorithm was used to determine the number of clusters for decreasing PCC values between 0.9 and 0.5 at 0.01 intervals (Figure 3D). The number of clusters increases steadily when lowering the PCC cutoff from 0.90 to approximately 0.70 after which it stabilizes between 0.72 and 0.60 and at lower thresholds even decreases. Combining the ranges of 0.60 to 0.72 and 0.70 to 0.82 made us choose the lowest overlapping cutoff of 0.70 for where biologically significant modules are most likely to be expected. We have not investigated networks of genes that are up-regulated in one set and down-regulated in the other (as would be represented by a negative PCC).

Using the PCC threshold of 0.70 a co-expression network was constructed and visualized with Cytoscape (Figure $3 \mathrm{E}$ ). The blue dots represent the selection of transcription factors and JAZ proteins having at least one edge (i.e. sharing at least one connection with other genes), and the colored dots represent the correspondingly colored genes from Figure 2. The total co-expression network thus obtained consists of 808 nodes that share 5951 edges. Statistical verification of our choice of cutoff by calculation of Bonferroni corrected p-values cannot be applied with data sets of this size, since cutoffs of as little as 0.2 can easily become statistically highly significant, while biological relevance at this low cutoff would be unlikely [28]. However, close co-expression of genes as deduced from our constructed network matched well with correlations found in literature (see below). Moreover, biochemical and functional analysis with gene sets selected from our network further supported its robustness [29].

\section{Exploration of Co-expressed Closest Neighbor Transcription Factors of Regulatory Genes}

The closest neighbors with a single edge distance from the regulatory genes shown in Figure 2 were separated in multiple sub cluster networks (Figures 4, 5, 6 and 7). The MAP kinase pathway from flagellin to defense genes (Figure 2, dark green boxes) is depicted in Figure 4A, and the MAP kinase pathway leading to the suppression of SA and induction of JA defense genes (Figure 2, purple boxes) is shown in Figure 4B. The network of genes co-expressed with the JA biosynthesis genes (Figure 2, yellow boxes) is depicted in Figure 5. Networks of ET biosynthesis (Figure 2, light blue hexagons) and ET signaling (Figure 2, pink ovals) are shown in Figures $6 \mathrm{~A}$ and $6 \mathrm{~B}$, respectively. Figure 7 shows the network of genes co-expressed with the genes leading to SA biosynthesis (Figure 2, red boxes). A detailed description of the above networks is given in the following paragraphs.

\section{The MAP Kinase Pathways}

The response to flagellin fragment flg22 as part of the PAMP signaling pathway is mediated via a MAPK

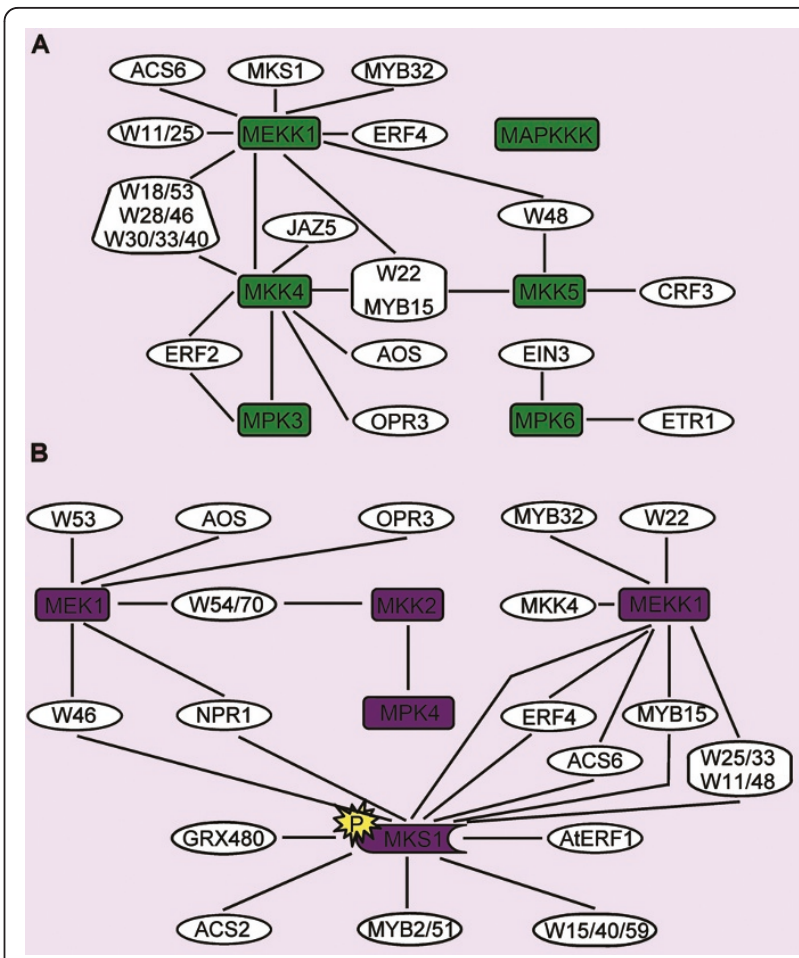

Figure 4 Co-expression network of the MAP kinase pathways. Co-expression network of MAP kinases leading to defense genes (A) and to SA defense gene repression and JA defense gene induction (B). The genes in colored boxes in the network correspond to similarly colored components of the signaling pathways indicated in Figure 2. The genes in white boxes indicate co-expressed genes with at least one edge to the kinase genes in the colored boxes.

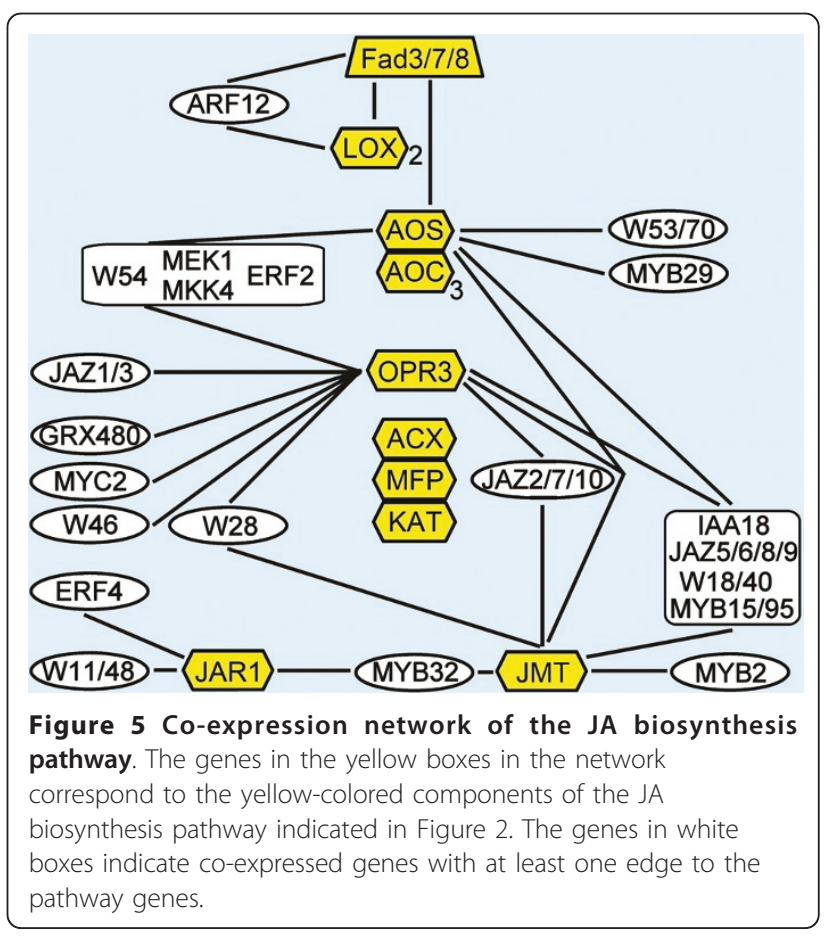




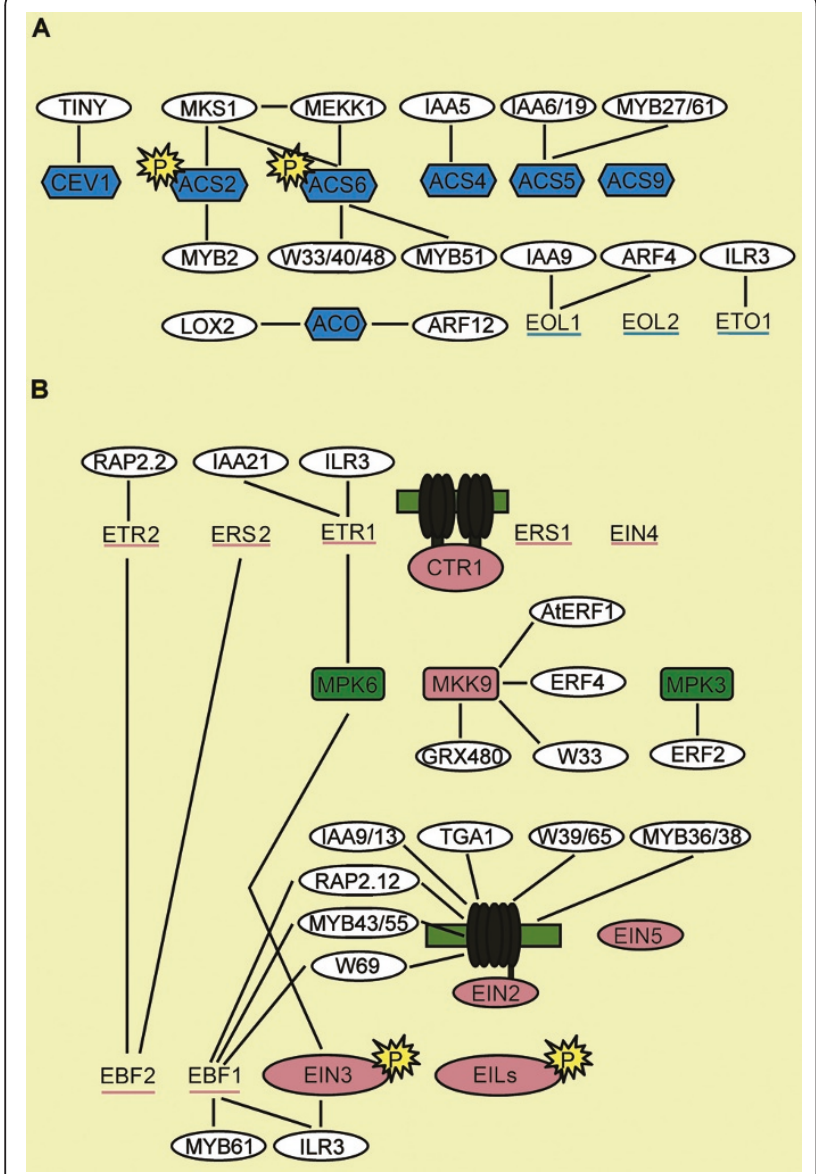

Figure 6 Co-expression network of the ET biosynthesis and signaling pathways. In panel $A$, the genes in the blue boxes in the network correspond to the blue-colored components of the ET biosynthesis pathway indicated in Figure 2. In panel B, The genes in colored boxes correspond to genes in similarly colored boxes of the ET signal transduction pathway shown in Figure 2. The genes in the white boxes in both panels indicate co-expressed genes having at least one edge to the pathway genes.

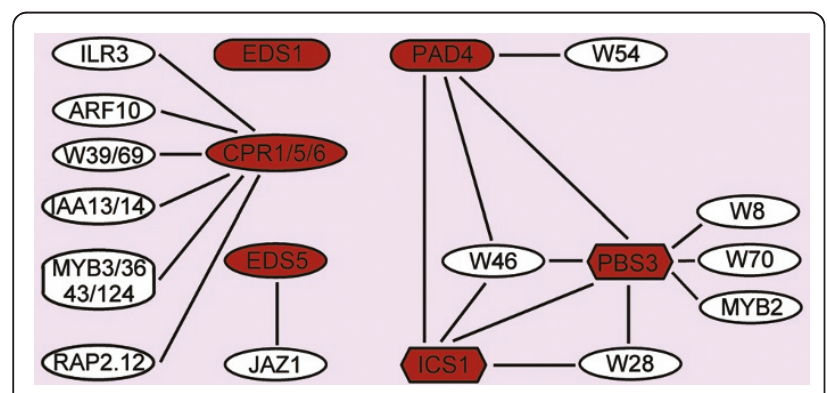

Figure 7 Co-expression network of the SA biosynthesis pathway. The genes in the red boxes in the network correspond to the red-colored components of the SA biosynthesis pathway indicated in Figure 2. The genes in white boxes indicate coexpressed genes with at least one edge to the pathway genes. cascade $[30,31]$. This signal transduction via MAPKKK/ MEKK1?-MKK4/MKK5-MPK3/MPK6 leads to transcriptional activation of downstream WRKY22 and WRKY29 genes, which results in the induction of resistance to both bacterial and fungal pathogens (Figure 2; [30]). Our results show that the genes encoding the MAPK components are highly co-expressed and form a network with a large number of co-expressed transcription factors (Figure 4A). The known downstream target of this cascade, WRKY22, is connected to MEKK1 and MKK4/MKK5. Surprisingly, MPK6 was not linked to any of the genes in the network, but appeared to be co-expressed with EIN3 and ETR1, both involved in the ET signaling pathway (Figure 4A; see below). As revealed by [32], multiple different models are possible of how MPK6 could be regulated directly under MEKK1. On the other hand, MPK6 has been described as the MAP kinase substrate of MKK3 and the MKK3-MPK6 cascade is important for the JA-dependent negative regulation of $M Y C 2$ [33]. MYC2 has the opposite effect on the MKK4/MPK3 branch. Induction of ERF2 activates a variety of wound response/insect resistance genes in JA-treated plants and regulates JA-dependent responses. ERF2 is positively regulated by $\mathrm{MYC} 2$ and in our analysis is connected to $M K K 4$ and MPK3[34,35]. Besides this connection, MKK4 is co-expressed with AOS and OPR3 (Figure 5) that are both important genes in the biosynthesis pathway of JA, suggesting that ERF2 might activate the MKK4/MPK3 cascade and via this route induce JA biosynthesis. With the biosynthesis of JA, in many cases also the JAZ repressor genes are positively regulated [36]. The connection between $M K K 4$ and JAZ5 might indicate that this branch is under control of the JAZ5 repressor.

The flagellin fragment flg22 not only affects the regulation of JA and ET pathways, but also activates the SA pathway. Many WRKY genes are co-expressed with MEKK1 and MKK4. WRKY28 is rapidly induced to very high levels upon flg22 treatment [37]. Together with WRKY28, WRKY46 is also co-regulated and they are both found as co-expressed genes with important genes in the SA biosynthesis pathway (Figure 7).

Both WRKY18 and WRKY53 are positive regulators of $P R$-gene expression and systemic acquired resistance (SAR). Functional WRKY18 is required for full induction of SAR and is linked to the activation of $P R-1$ [38]. WRKY18 enhances resistance against Pseudomonas syringae [39]. The link between WRKY53 and MEK1 can be explained via MEKK1 (Figure 4B). MEKK1 is upstream of MEK1 and interacts with an activation domain protein that can be phosphorylated and binds to the promoter of WRKY53 to activate gene expression [40]. This links WRKY18 and WRKY53 to flg22 and the initiation of SAR mediated defense within our coexpression network. 
The MAPK cascade (MEKK1-MEK1/MKK2-MPK4), induced by challenge inoculation with $P$. syringae or treatment with flg22, leads to phosphorylation of MAP kinase substrate 1 (MKS1), which forms a complex with MPK4 and WRKY33 and possibly WRKY25. Upon phosphorylation of MKS1, WRKY33 is released inthe nucleus to initiate positive regulation of JAinduced defense genes and negative regulation of SArelated defense genes. Also other WRKYs, like WRKY11 and WRKY17, act as negative regulators of basal resistance responses [41-44]. Almost all of the genes encoding these WRKYs were found interconnected in the co-expression network (Figure 4B). WRKY48 is also stress and pathogen inducible and acts as a transcription factor that represses basal defense and PR-gene expression. When considering its location in the co-expression network, WRKY48 could function in a similar manner as WRKY11/17 and/or WRKY25/ 33 [45].

WRKY70 and the functional homolog WRKY54 have dual roles in SA-mediated gene expression and resistance. Upon high accumulation of SA, WRKY54/70 act as negative regulators of SA biosynthesis. Besides this negative role, they activate other SA-regulated genes $[38,46]$. The route via which WRKY54 and WRKY70 repress SA biosynthesis is unknown. Within the coexpression network both these WRKYs link to both $M E K 1$ and $M K K 2$, two important kinases in the cascade that leads to repression of SA defense genes. It may be that negative regulation of SA biosynthesis is brought about by activation of this MAP kinase cascade by WRKY54 and WRKY70.

\section{The JA Biosynthesis Pathway}

The JAZ repressor proteins play an important role in JA signaling. The initial JAZ repressor that is released from MYC2 to activate transcription of target genes is JAZ3 $[36,47] . M Y C 2, J A Z 1$ and $J A Z 3$ are directly linked in the co-expression network with OPR3, encoding 12-oxophytodienoate reductase, an essential enzyme in JA biosynthesis (Figure 5). Several other genes encoding JAZ repressors are also connected to OPR3 and to the gene encoding $J A$ methyl transferase (JMT), while others link to both JMT and the gene for allene oxide synthase $(A O S)$. The various connections of these $J A Z$ genes may hint at which levels the different JAZ repressors are operational (Figure 5).

Surprisingly, many of the WRKY transcription factors that are involved in positive or negative regulation of PR-genes and SAR are also connected to the JA biosynthesis pathway (Figure 5 ), like the positive regulatory combinations WRKY18/53 (Figure 4A), WRKY54/70 (Figure 4B), WRKY28/46 that are possibly involved in the regulation of SA biosynthesis (Figure 7) and
WRKY11/48 that act as negative regulators of SA defense genes.

Several members of the MYB transcription factor family were also found to be closely co-expressed with the JA biosynthesis genes AOS, OPR3 and JMT. Most of the co-expressed MYB transcription factors have no known function. Using publicly available online coexpression analyses, a link was found between MYB29 and the regulation of aliphatic glucosinolate biosynthesis [25]. Since methyl-JA is involved in regulation of glucosinolate biosynthesis this could indicate that MYB29 is co-expressed at the level of JMT or below. However, the upstream connection of MYB29 with AOS suggests that activation of the glucosinolate pathway by MYB29 is already initiated before methyl-JA is synthesized.

\section{The ET Biosynthesis and Signaling Pathway}

ET is produced from S-adenosyl-methionine in a twostep reaction catalyzed by the enzymes aminocyclopropane carboxylic acid (ACC)-synthase (encoded by $A C S$ genes) and ACC-oxidase (encoded by $A C O$ ), respectively. Genes co-expressed with the ET biosynthesis genes are depicted in Figure 6A. We found a connection between $A C S 2 / 6$ and $M E K K 1 / M K S 1$ of the MAP kinase pathway. MEKK1 has been proposed to lead to phosphorylation of MPK6, although the mechanism through which this might occur has not yet been established. Different models for this regulation have been proposed [32]. ACS2 and ACS6 can be phosphorylated by MPK6 (Figure 2). This phosphorylation stabilizes the protein, which results in increased ET production [48]. Other genes co-expressed with the ET biosynthesis genes ACS4, ACS5 and ACO encode a variety of Aux/IAA and ARF factors. In a review by Reed [49] it is proposed that targets of Aux/IAA and ARF might include genes encoding ACC synthase. Various other Aux/IAA and ARF genes were found to be closely co-expressed with a number of other regulator genes (encoding ubiquitin ligases EOL1, ETO1) involved in ET biosynthesis, indicative of a possible function in the integration of ET and auxin signaling pathways.

The MAP kinases in the ET signaling pathway (Figure $6 \mathrm{~B})$ are connected to a limited number of other nodes. The link between MPK3 and ERF2 was discussed above. Mutant studies with the etr1-1 gain-of-function ETinsensitive mutant placed MPK6 directly downstream of ETR1 [50,51]. This is also observed within the coexpression network. In the network EIN3 is also connected to MPK6. In the MKK9-MPK3/6 cascade it was shown that direct phosphorylation in the nucleus via this cascade stabilizes the EIN3 protein, which may be a key step in ET signaling (Figure 2; [52]). The involvement of MKK 9 at this point of the pathway has recently been questioned [53]. Notably, in the co-expression 
network MKK9 doesn't correlate with any genes known to be involved at this point of the pathway, further undercutting the suggested involvement of MKK9 in ET-signaling. Within the co-expression network depicted in Figure 3E both genes for ETR1 and MPK6 (represented by the pink and green dot almost in the middle of the network), are in between the super cluster with the genes encoding proteins involved in SA signaling (red dots), Flg22-initiated MAPK kinase cascade (green dots) and the JA biosynthesis genes (yellow dots), and the super cluster with several genes involved in the ET signaling pathway (pink dots). The central location of MPK6 and ETR1 between the super clusters with the other signaling genes might be indicative for a role of the combination of ETR1/MPK6 in crosstalk between these clusters.

Within the ethylene-signaling network (Figure 6B) we found many genes co-expressed with EIN2. For almost none of these genes a clear function has been described in literature so far. Recently, it was found that the modulation of NPR1 dependency of SA-JA crosstalk by ET is dependent on EIN2 [54]. Most of the genes involved in the crosstalk have not yet been assigned to a particular function. Surprisingly, in our analysis many of the genes that are co-expressed with EIN2 (IAA13, RAP2.12, MYB36, MYB43, WRKY39, WRKY69) are also connected to CPR5 in the SA biosynthesis pathway (see below). It is tempting to assume that some of these genes are involved in the EIN2-dependent crosstalk with SA.

\section{The SA Biosynthesis Pathway}

Heterodimerization of EDS1 and PAD4 and their nuclear localization are important for subsequent steps in the SA signaling pathway [55]. Recently, it was found that EDS1 expression is repressed by the $\mathrm{Ca}^{2+} /$ calmodulin-binding transcription factor AtSR1, indicating that SA levels are regulated by $\mathrm{Ca}^{2+}[56]$. We found that the gene encoding the $\mathrm{Ca}^{2+}$ /calmodulin-binding transcription factor MYB2, is co-expressed with PBS3 (Figure 7; [57]). If MYB2, like AtSR1, acts as a repressor of SA accumulation, this might indicate another point of regulation. In addition to the link to $P B S 3, M Y B 2$ is also connected to $J M T$ in the methyl-JA synthesis pathway and to ACS2 in the ET biosynthesis pathway, suggestive for a role of MYB2 in fine-tuning SA, JA, and ET biosynthesis. Besides the connections of WRKY54 and WRKY70 that are already known to influence biosynthesis of SA, we found two new WRKY genes (WRKY28 and WRKY46) to be co-expressed with isochorismate synthase 1 (ICS1), a key enzyme in the biosynthesis of SA. As described above, WRKY28 is known to be rapidly induced by flg22, while WRKY46 is rapidly induced downstream of avirulence effectors [58]. This might indicate a direct role for these WRKYs in flagellin and avirulence effector-induced biosynthesis of SA. Another WRKY gene that we found to be co-expressed with PBS3 is WRKY8. This WRKY is described in literature as one that is evolutionary highly related to WRKY28 [59].

To illustrate the validity of our choice to limit the coexpression analysis to the set of stress-related microarrays, in Figure 8 we focused on the sub network around ICS1/PBS3. In Figure 8A, all genes that were found coexpressed in the stress-related set within one edge at the PCC cutoff of 0.7 are displayed. Among the co-expressed genes are WRKY7O and PAD4, which are proven factors in the SA-signaling pathway $[38,55]$. This sub-network degraded when only the set of development-related genes (Figure 8B) or the set of all 1436 available micro-arrays

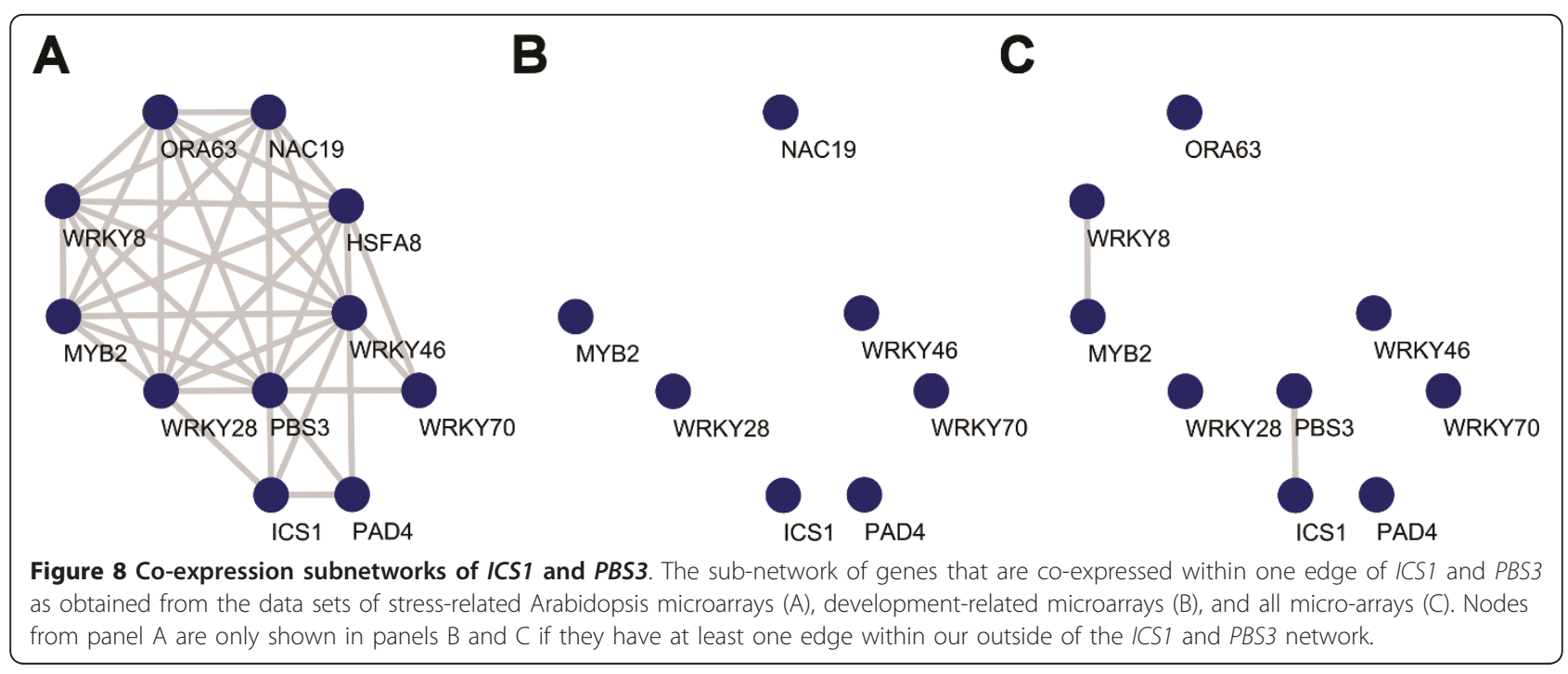


were considered (Figure 8C). This supports the notion that also other genes in the dataset may play roles in the stress-related pathways investigated. Based on the results of the co-expression sub-network around ICS1 and PBS3, in a follow-up paper we investigated the possible role of transcription factors WRKY28 and WRKY46 in ICS1 and PBS3 gene expression [29].

In Figures 4, 5, 6 and 7 only co-expressed, established transcriptional regulators are depicted. A full list of all genes found to be closely co-expressed with the pathway components in Figure 2 is given in Additional file 2, Table 2.

\section{Conclusions}

Our study shows that co-expression analysis using a selection of publicly available stress related data sets resulted in many new, potential components of the signal transduction pathways involved in stress responses. This could aid in the further characterization of these pathways.

\section{Methods}

\section{Microarray Dataset}

The dataset of 1436 Affymetrix Arabidopsis 25K arrays obtained from NASCArrays and AtGenExpress was downloaded from ftp.arabidopsis.org. This dataset has already been normalized using the robust multi-array method (RMA). For tracking down the experimental conditions of the different arrays we used the mapping file provided and with assistance from the curators of TAIR the codes were converted into matching experimental conditions that can be found on the website. Based on these experimental conditions selections were made of stress- and development-related datasets that were used in our experiments.

\section{Bi-clustering, Pearson Cutoff Determination and Co- expression Analysis}

For the bi-clustering the raw RMA normalized expression values were transformed such, to obtain mean expression values of 0 and a standard deviation of 1 for all rows. Clustering of the data was performed using the following parameters: the distance between objects in the data matrix was one minus the sample correlation between points (treated as sequences of values), linkage was set to complete (furthest distance), and the cutoff within the dendogram for the hierarchical cluster tree was set to 0.50 . All values below this cutoff were given a different color for both the experimental conditions and the genes.

To determine a biologically relevant Pearson correlation cutoff, the number of nodes and edges and the network density were determined using the raw RMA normalized expression values for different PCC cutoffs ranging from 0 to 1 at 0.01 intervals per data point using the 372 microarrays from the selected set of stress-related micro-arrays. The total number of clusters was determined using the MCODE algorithm within Cytoscape for PCC cutoffs from 0.5 to 0.9 at 0.01 intervals using the following settings: loops not included, degree cutoff $=2$, Haircut on, fluff off, node score cutoff $=0.2, \mathrm{~K}$-score $=2$, Max depth $=100$.

The co-expression network was built using the raw RMA normalized expression values with a PCC cutoff of 0.70 for the stress dataset and was visualized using Cytoscape using standard settings.

\section{Additional material}

Additional file 1: Table 1. Lists of the 372 microarrays and the genes used for analysis in combination with Figure 2.

Additional file 2: Table 2. Genes encoding transcriptional regulators closely co-expressed with signaling pathway genes.
Acknowledgements and Funding

We would like to thank the curators of The Arabidopsis Information Resource (TAIR) for helpful suggestions for tracking the experimental conditions of most of the micro-arrays in the dataset. The work was performed without external funding.

\section{Authors' contributions}

MW designed the study, carried out the analysis, helped in data interpretation, and made the draft of the manuscript. JFB and HL helped in data interpretation and edited the manuscript. All authors have given final approval for this version to be published.

Received: 22 December 2010 Accepted: 19 May 2011 Published: 19 May 2011

\section{References}

1. Agarwal PK, Jha B: Transcription factors in plants and ABA dependent and independent abiotic stress signalling. Biologia Plantarum 2010, 54:201-212.

2. Bari $R$, Jones JDG: Role of plant hormones in plant defence responses. Plant Molecular Biology 2009, 69:473-488.

3. Pieterse CMJ, Leon-Reyes A, Van der Ent S, Van Wees SCM: Networking by small-molecules hormones in plant immunity. Nat Chem Biol 2009, 5:308-316.

4. van Verk MC, Gatz C, Linthorst HJM: Transcriptional Regulation of Plant Defense Responses. In Adv Bot Res. Volume 51. Edited by: van Loon LC. Elsevier; 2009:397-438.

5. Ramirez V, Coego A, Lopez A, Agorio A, Flors V, Vera P: Drought tolerance in Arabidopsis is controlled by the OCP3 disease resistance regulator. The Plant Journal 2009, 58:578-591.

6. Yasuda M, Ishikawa A, Jikumaru Y, Seki M, Umezawa T, Asami T, MaruyamaNakashita A, Kudo T, Shinozaki K, Yoshida S, Nakashita H: Antagonistic interaction between systemic acquired resistance and the abscisic acidmediated abiotic stress response in Arabidopsis. The Plant Cell 2008, 20:1678-1692.

7. Czechowski T, Bari RP, Stitt M, Scheible W, Udvardi MK: Real-time RT-PCR profiling of over 1400 Arabidopsis transcription factors: unprecedented sensitivity reveals novel root- and shoot-specific gene. The Plant Journal 2004, 38:366-379.

8. Aoki K, Ogata Y, Shibata D: Approaches for Extracting Practical Information from Gene Co-expression Networks in Plant Biology. Plant Cell Physiol 2007, 48:381-390. 
9. Edgar R, Domrachev M, Lash AE: Gene Expression Omnibus: NCBI gene expression and hybridization array data repository. Nucleic Acids Res 2002, 30:207-210.

10. Brazma A, Parkinson H, Sarkans U, Shojatalab M, Vilo J, Abeygunawardena N, Holloway E, Kapushesky M, Kemmeren P, Lara GG, Oezcimen A, Rocca-Serra P, Sansone SA: ArrayExpress - a public repository for microarray gene expression data at the EBI. Nucleic Acids Res 2003 , 31:68-71.

11. Steinhauser D, Usadel B, Luedemann A, Thimm O, Kopka J: CSB.DB: a comprehensive systems-biology database. Bioinformatics 2004, 20:3647-3651

12. Zimmermann P, Hirsch-Hoffmann M, Hennig L, Gruissem W: GENEVESTIGATOR: Arabidopsis Microarray Database and Analysis Toolbox. Plant Physiology 2004, 136:2621-2632.

13. Zimmermann P, Hennig L, Gruissem W: Gene expression analysis and network discovery using Genevestigator. Trends in Plant Science 2005, 10:407-409.

14. Hruz T, Laule O, Szabo G, Wessendrop F, Bleuler S, Oertle L, Widmayer P, Gruissem W, Zimmermann P: Genevestigator V3: A reference expression database for the meta-analysis of transcriptomes. Advances in Bioinformatics 2008, Article ID 420747

15. Toufighi K, Brady SM, Austin R, Ly E, Provart NJ: The Botany Array Resource: e-Northerns, Expression Angling, and promoter analyses. The Plant Journal 2005, 43:153-163.

16. Manfield IW, Jen CH, Pinney JW, Michalopoulos I, Bradford JR, Gilmartin PM, Westhead DR: Arabidopsis Co-expression Tool (ACT): web server tools for microarray-based gene expression analysis. Nucleic Acids Res 2006, 34 W504-509.

17. Obayashi T, Kinoshita K, Nakai K, Shibaoka M, Hayashi S, Saeki M, Shibata D, Saito K, Ohta H: ATTED-II: a database of co-expressed genes and cis elements for identifying co-regulated gene groups in Arabidopsis. Nucleic Acids Res 2007, 35:D863-D869.

18. Obayashi T, Hayashi S, Shibaoka M, Saeki M, Ohta H, Kinoshita K: COXPRESdb: a database of coexpressed gene networks in mammals. Nucleic Acids Res 2008, 36:D77-82

19. Obayashi T, Hayashi S, Saeki M, Ohta H, Kinoshita K: ATTED-II provides coexpressed gene networks for Arabidopsis. Nucleic Acids Res 2009, 37 D987-991.

20. Akiyama K, Chikayama E, Yuasa H, Shimada Y, Tohge T, Shinozaki K, Hirai MY, Sakurai T, Kikuchi J, Saito K: PRIMe: A Web site that assembles tools for metabolomics and transcriptomics. In Silico Biol 2008, 8:0027.

21. Srinivasasainagendra V, Page GP, Mehta T, Coulibaly I, Loraine AE: CressExpress: a tool for large-scale mining of expression data from Arabidopsis. Plant Physiology 2008, 147:1004-1016.

22. Birnbaum K, Shasha DE, Wang JY, Jung JW, Lambert GM, Galbraith DW, Benfey PN: A gene expression map of the Arabidopsis root. Science 2003, 302:1956-1960.

23. Elo A, Lyznik A, Gonzalez DO, Kachman SD, Mackenzie SA: Nuclear genes that encode mitochondrial proteins for DNA and RNA metabolism are clustered in the Arabidopsis genome. The Plant Cell 2003, 15:1619-1631.

24. Persson S, Wei H, Milne J, Page GP, Somerville CR: Identification of genes required for cellulose synthesis by regression analysis of public microarray data sets. Proceedings of the National Academy of Sciences of the United States of America 2005, 102:8633-8638.

25. Hirai MY, Sugiyama K, Sawada Y, Tohge T, Obayashi T, Suzuki A, Araki R, Sakurai N, Suzuki H, Aoki K, Goda H, Nishizawa Ol, Shibate D, Saito K: Omics-based identification of Arabidopsis Myb transcription factors regulating aliphatic glucosinolate biosynthesis. Proceedings of the National Academy of Sciences of the United States of America 2007, 104:6478-6483.

26. Ma S, Gong Q, Bohnert HJ: An Arabidopsis gene network based on the graphical Gaussian model. Genome Res 2007, 17:1614-1625.

27. Borate BR, Chesler EJ, Langston MA, Saxton AM, Voy BH: Comparison of threshold selection methods for microarray gene co-expression matrices. BMC Res Notes 2009, 2:240.

28. Usadel B, Obayashi T, Mutwil M, Giorgi FM, Bassel G, Tanimoto M, Chow A, Steinhauser D, Persson S, Provart NJ: Co-expression tools for plant biology: opportunities for hypothesis generation and caveats. Plant, Cell and Environment 2009, 32:1633-1651.

29. van Verk MC, Bol JF, Linthorst HJM: WRKY Transcription Factors Involved in Activation of SA Biosynthesis Genes. BMC Plant Biol 2011, 11:89.
30. Asai T, Tena G, Plotnikova J, Willmann MR, Chiu WL, Gomez-Gomez L, Boller T, Ausubel FM, Sheen J: MAP kinase signalling cascade in Arabidopsis innate immunity. Nature 2002, 415:977-983.

31. Suarez-Rodriguez MC, Adams-Phillips L, Liu Y, Wang H, Su SH, Jester PJ, Zhang S, Bent AF, Krysan PJ: MEKK1 is required for flg22-induced MPK4 activation in Arabidopsis plants. Plant Physiology 2007, 143:661-669.

32. Mészáros $T$, Helfer $A$, Bögre $L$ : The more we know, the less we understand? Complexity of MAP kinase signaling. Plant Signal Behav 2007, 2:30-32.

33. Takahashia F, Yoshidab R, Ichimurac K, Mizoguchib T, Seoe S, Yonezawac M, Maruyamaf K, Yamaguchi-Shinozakif K, Shinozakia K: The MitogenActivated Protein Kinase Cascade MKK3-MPK6 Is an Important Part of the Jasmonate Signal Transduction Pathway in Arabidopsis. The Plant Cell 2007, 19:805-818.

34. McGrath KC, Dombrecht B, Manners JM, Schenk PM, Edgar Cl, Maclean DJ, Scheible WR, Udvardi MK, Kazan K: Repressor- and activator-type ethylene response factors functioning in jasmonate signaling and disease resistance identified via a genome-wide screen of Arabidopsis transcription factor gene expression. Plant Physiology 2005, 139:949-959.

35. Dombrecht B, Xue GP, Sprague SJ, Kirkegaard JA, Ross JJ, Reid JB, Fitt GP, Sewelam N, Schenk PM, Manners JM, Kazana K: MYC2 differentially modulates diverse jasmonate-dependent functions in Arabidopsis. The Plant Cell 2007, 19:2225-2245.

36. Chini A, Fonseca S, Fernández G, Adie B, Chico JM, Lorenzo O, GarcíaCasado G, López-Vidriero I, Lozano FM, Ponce MR, Micol JL, Solano R: The JAZ family of repressors is the missing link in jasmonate signalling Nature 2007, 448:666-671.

37. Navarro L, Zipfel C, Rowland O, Keller I, Robatzek S, Boller T, Jones JDG: The transcriptional innate immune response to flg22. Interplay and overlap with Avr gene-dependent defense responses and bacterial pathogenesis. Plant Physiology 2004, 135:1113-1128.

38. Wang D, Amornsiripanitch N, Dong X: A genomic approach to identify regulatory nodes in the transcriptional network of systemic acquired resistance in plants. PLOS Pathogens 2006, 2:e123.

39. Xu X, Chen C, Fan B, Chen Z: Physical and functional interactions between pathogen-induced Arabidopsis WRKY18, WRKY40, and WRKY60 transcription factors. The Plant Cell 2006, 18:1310-1326.

40. Miao Y, Laun TM, Smykowski A, Zentgraf U: Arabidopsis MEKK1 can take a short cut: it can directly interact with senescence-related WRKY53 transcription factor on the protein level and can bind to its promoter. Plant Molecular Biology 2007, 65:63-76.

41. Andreasson $E$, Jenkins $T$, Brodersen $P$, Thorgrimsen $S$, Petersen NHT, Zhu $S$ Qiu JL, Micheelsen P, Rocher A, Petersen M, Newman MA, Nielsen HB, Hirt H, Somssich I, Mattsson O, Mundy J: The MAP kinase substrate MKS1 is a regulator of plant defense responses. The EMBO Journal 2005, 24:2579-2589.

42. Brodersen $P$, Petersen M, Nielsen HB, Zhu S, Newman MA, Shokat KM, Rietz S, Parker J, Mundy J: Arabidopsis MAP kinase 4 regulates salicylic acid- and jasmonic acid/ethylene-dependent responses via EDS1 and PAD4. The Plant Journal 2006, 47:532-546.

43. Journot-Catalino N, Somssich IE, Roby D, Kroj T: The transcription factors WRKY11 and WRKY17 act as negative regulators of basal resistance in Arabidopsis thaliana. The Plant Cell 2006, 18:3289-3302.

44. Qiu JL, Zhou L, Yun BW, Nielsen HB, Fiil BK, Petersen K, Mackinlay J, Loake GJ, Mundy J, Morris PC: Arabidopsis mitogen-activated protein kinase kinases MKK1 and MKK2 have overlapping functions in defense signaling mediated by MEKK1, MPK4, and MKS1. Plant Physiology 2008, 148:212-222.

45. Xing DH, Laia ZB, Zhenga ZY, Vinoda KM, Fana BF, Chen ZX: Stress- and Pathogen-Induced Arabidopsis WRKY48 is a Transcriptional Activator that Represses Plant Basal Defense. Mol Plant 2008, 1:459-470.

46. Kalde M, Barth M, Somssich IE, Lippok B: Members of the Arabidopsis WRKY group III transcription factors are part of different plant defense signaling pathways. Molecular Plant-Microbe Interactions 2003, 16:295-305.

47. Thines B, Katsir L, Melotto M, Niu Y, Mandaokar A, Liu G, Nomura K, He SY, Howe GA, Browse J: JAZ repressor proteins are targets of the SCF ${ }^{\mathrm{CO} 11}$ complex during jasmonate signalling. Nature 2007, 448:661-665.

48. Liu Y, Zhang S: Phosphorylation of 1-aminocyclopropane-1-carboxylic acid synthase by MPK6, a stress-responsive mitogen-activated protein kinase, induces ethylene biosynthesis in Arabidopsis. The Plant Cell 2004, 16:3386-3399. 
49. Reed JW: Roles and activities of Aux/IAA proteins in Arabidopsis. Trends in Plant Science 2001, 6:420-425.

50. Chang C: Ethylene signaling: the MAPK module has finally landed. Trends in Plant Science 2003, 8:365-368.

51. Ouaked F, Rozhon W, Lecourieux D, Hirt H: A MAPK pathway mediates ethylene signaling in plants. The EMBO Journal 2003, 22:1282-1288.

52. Yoo SD, Cho YH, Tena G, Xiong Y, Sheen J: Dual control of nuclear EIN3 by bifurcate MAPK cascades in $\mathrm{C}_{2} \mathrm{H}_{4}$ signalling. Nature 2008, 451:789-795.

53. An F, Zhao Q, Ji Y, Li W, Jiang Z, Yu X, Zhang X, Zhang C, Han Y, He W, Liu Y, Zhang S, Ecker JR, Guo H: Ethylene-Induced stabilization of ETHYLENE INSENSITIVE3 and EIN3-LIKE1 is mediated by proteasomal degradation of EIN3 binding F-Box 1 and 2 that requires EIN2 in Arabidopsis. The Plant Cell 2010, 22:2384-2401.

54. Leon-Reyes A, Spoel SH, De Lange ES, Abe H, Kobayashi M, Tsuda S, Millenaar FF, Welschen RAM, Ritsema T, Pieterse CMJ: Ethylene modulates the role of NONEXPRESSOR OF PATHOGENESIS-RELATED GENES1 in cross talk between salicylate and jasmonate signaling. Plant Physiology 2009, 149:1797-1809.

55. Feys BJ, Moisan $L$, Newman MA, Parker JE: Direct interaction between the Arabidopsis disease resistance signaling proteins, EDS1 and PAD4. The EMBO Journal 2001, 20:5400-5411.

56. Du L, Ali GS, Simons KA, Hou J, Yang T, Reddy ASN, Poovaiah BW: Ca2 ${ }^{+} /$calmodulin regulates salicylic-acid-mediated plant immunity. Nature 2009, 457:1154-1158.

57. Yoo JH, Park CY, Kim JC, Heo WD, Cheong MS, Park HC, Kim MC, Moon BC, Choi MS, Kang YH, Lee JH, Kim HS, Lee SM, Yoon HW, Lim CO, Yun DJ, Lee SY, Chung WS, Cho MJ: Direct Interaction of a Divergent CaM Isoform and the Transcription Factor, MYB2, Enhances Salt Tolerance in Arabidopsis. Journal of Biological Chemistry 2005, 280:3697-3706.

58. He P, Shan L, Lin NC, Martin GB, Kemmerling B, Nürnberger T, Sheen J: Specific bacterial suppressors of PAMP signaling upstream of MAPKKK in Arabidopsis innate immunity. Cell 2006, 125:563-575.

59. Yamasaki K, Kigawa T, Inoue M, Tateno M, Yamasaki T, Yabuki T, Aoki M, Seki E, Matsuda T, Tomo Y, Hayami N, Terada T, Shirouzu M, Tanaka A, Seki M, Shinozaki K, Yokoyama S: Solution structure of an Arabidopsis WRKY DNA binding domain. The Plant Cell 2005, 17:944-956.

doi:10.1186/1471-2229-11-88

Cite this article as: van Verk et al:: Prospecting for Genes involved in transcriptional regulation of plant defenses, a bioinformatics approach. BMC Plant Biology 2011 11:88.

\section{Submit your next manuscript to BioMed Central and take full advantage of:}

- Convenient online submission

- Thorough peer review

- No space constraints or color figure charges

- Immediate publication on acceptance

- Inclusion in PubMed, CAS, Scopus and Google Scholar

- Research which is freely available for redistribution

Submit your manuscript at www.biomedcentral.com/submit 